\title{
A Fatigue Strength Predictor for Steels Using Ensemble Data Mining
}

\author{
Ankit Agrawal and Alok Choudhary \\ Electrical Engineering and Computer Science \\ Northwestern University \\ Evanston IL 60201
}

\begin{abstract}
Fatigue strength is one of the most important mechanical properties of steel. High cost and time for fatigue testing, and potentially disastrous consequences of fatigue failures motivates the development of predictive models for this property. We have developed advanced data-driven ensemble predictive models for this purpose with an extremely high cross-validated accuracy of $>98 \%$, and have deployed these models in a user-friendly online web-tool, which can make very fast predictions of fatigue strength for a given steel represented by its composition and processing information. Such a tool with fast and accurate models is expected to be a very useful resource for the materials science researchers and practitioners to assist in their search for new and improved quality steels. The web-tool is available at http://info.eecs.northwestern.edu/SteelFatigueStrengthPredictor
\end{abstract}

\section{INTRODUCTION}

Deciphering the processing-structure-property-performance (PSPP) relationships in materials is the key to understand the science of materials, and subsequently engineer and design new improved materials. The Materials Genome Initiative (MGI) was launched by the US government in 2011 [1] to realize the vision of development of advanced materials necessary for economic security and human well-being. The MGI Strategic Plan released in 2014 [2] also identifies data analytics as one of the key objectives as part of integrating experiments, computation, and theory, in order to realize the vision of MGI. The increasing applications of data analytics in this domain has made the new field of materials informatics very popular [4].

It is in the spirit and pursuit of the vision and approach of MGI that we discuss and present in this demonstration paper, an online data informatics tool deploying forward PSPP models to predict the fatigue strength of a given steel alloy, given its composition and processing. Fatigue strength of steel is a crucial property to know, given the high cost and time of fatigue testing, and potentially disastrous con-

Permission to make digital or hard copies of part or all of this work for personal or classroom use is granted without fee provided that copies are not made or distributed for profit or commercial advantage and that copies bear this notice and the full citation on the first page. Copyrights for third-party components of this work must be honored. For all other uses, contact the owner/author(s).

CIKM'16 October 24-28, 2016, Indianapolis, IN, USA

(C) 2016 Copyright held by the owner/author(s)

ACM ISBN 978-1-4503-4073-1/16/10

DOI: http://dx.doi.org/10.1145/2983323.2983343 sequences of fatigue failures. It is the most important information required for design and failure analysis of mechanical components. Fatigue is estimated to account for over $90 \%$ of all mechanical failures of structural components, and hence, fatigue strength prediction is of critical importance. The predictive models deployed in the tool are a result of the application of supervised learning techniques on an experimental fatigue dataset from Japan National Institute of Materials Science MatNavi database [3], which is freely accessible online. This dataset has been previously used to perform similar predictive modeling $[6,5]$, but the resulting models had not been deployed. Following are the contributions of this study:

- Comparison of 40 supervised modeling configurations on the NIMS steel fatigue dataset, including ensemble modeling techniques, resulting in more accurate predictive models than prior works on the same data.

- Using data-driven feature selection techniques to identify a reduced set of non-redundant attributes for the purpose of building a predictive model using fewer input features while still having a satisfactory predictive accuracy.

- Deployment of the most accurate "forward" PSPP models using both the full and reduced set of attributes in a publicly accessible web-tool.

\section{DATASET}

The overall data-driven analytics process is depicted as a block diagram in Figure 1. Rotating bending fatigue testing data was obtained from the publicly available NIMS MatNavi database [3]. This NIMS data has 437 experimental observations of steel, with 9 composition attributes $(\% \mathrm{C}$, $\% \mathrm{Si}, \% \mathrm{Mn}, \% \mathrm{P}, \% \mathrm{~S}, \% \mathrm{Ni}, \% \mathrm{Cr}, \% \mathrm{Cu}$, and $\% \mathrm{Mo}$ (remaining is $\mathrm{Fe})$ ), 16 processing attributes (normalization temperature, through hardening temperature, through hardening time, cooling rate for through hardening, carburization temperature, carburization time, diffusion temperature, diffusion time, quenching media temperature, tempering temperature, tempering time, cooling rate for tempering, reduction ratio, area proportion of inclusions deformed by plastic work, those occurring in discontinuous array, and the isolated ones), and 1 mechanical property (rotating bending fatigue strength @ $10^{7}$ cycles.) The work in [5] preprocessed this data to make a coherent database with all key processes included (for cases where a process did not take place, the time was set to zero and temperature to austenization temperature or average of rest of the data). This data was made 


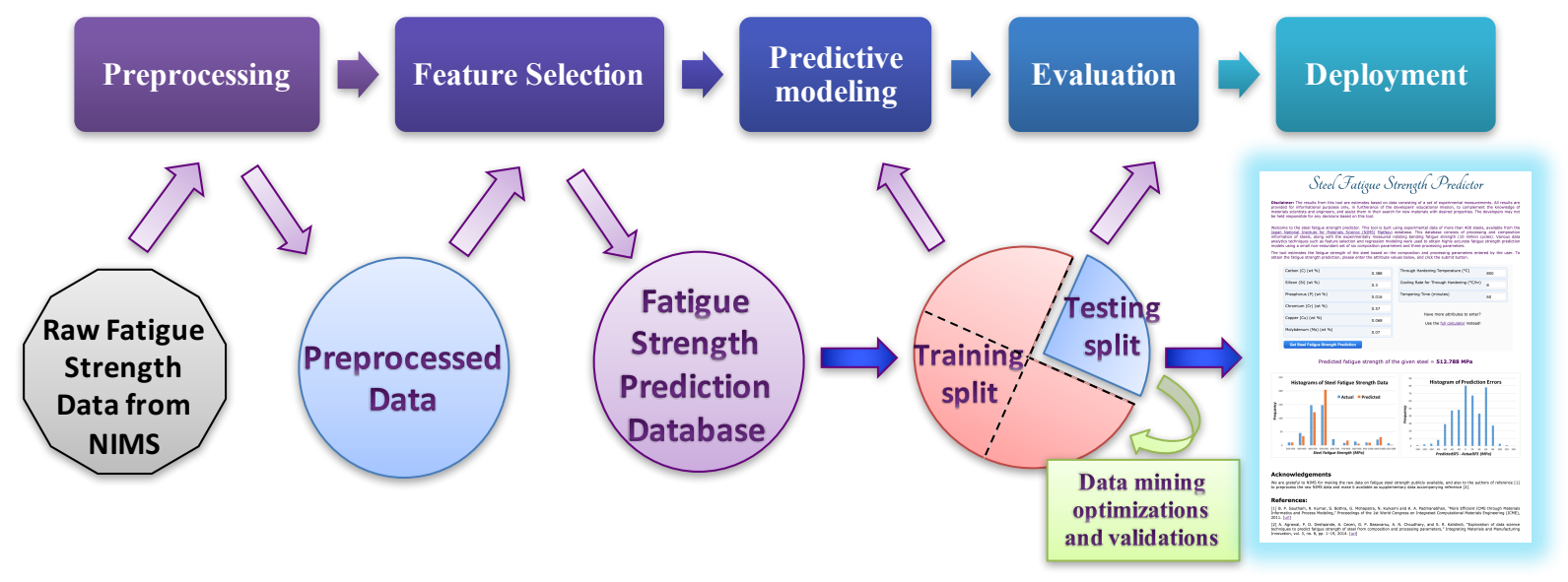

Figure 1: The data mining workflow used in this work.

available as supplementary data of [5], and is the starting point of the current study.

\section{EXPERIMENTS AND RESULTS}

We used 40 regression schemes in this study listed in Table 1 , including both direct application of regression techniques and constructing their ensembles using various ensembling techniques (compatible combinations). The correlation feature selection (CFS) method was used for feature selection. It is used to identify a subset of features highly correlated with the class variable and weakly correlated amongst them. CFS was used in conjunction with a best first search to find a subset with best average merit. WEKA software [7] version 3.7.13 was used for all analytics with default parameters, unless otherwise stated. Quantitative assessments of the degree to how close the models could predict the experimental fatigue strength are used to evaluate the models' predictive performance. The metrics used for this purpose include the coefficient of correlation $(R)$, explained variance $\left(R^{2}\right)$, Mean Absolute Error $(M A E)$, Root Mean Squared Error $(R M S E)$, Relative Absolute Error $(R A E)$, and Root Relative Squared Error (RRSE).

Table 2 presents the comparison of the top 10 modeling techniques used in this work with respect to the different metrics mentioned earlier. All results are based on 10 independent runs of 10 -fold cross-validation to facilitate statistical significance testing. In addition, the training and testing times for each model, and the model size is also listed. The top four models from Table 2 are subsequently combined using the Voting modeling scheme to obtain the final model $\left(R^{2}=0.9819, M A E=17.67 M P a, R M S E=25.08 M P a\right)$, whose performance was found to be significantly better than all the four constituent models, as well as better than the modeling techniques used previously in [5], at $p=0.05$. Visual inspection of the scatter plots (Figure 2) also reveal that the current model is able to make more accurate predictions for carbon and low alloy steels, where the best model in [5] had failed.

The application of CFS technique to composition attributes identified a subset of six composition attributes: C, Si, P, $\mathrm{Cr}, \mathrm{Cu}$, and Mo. The same analysis on processing attributes

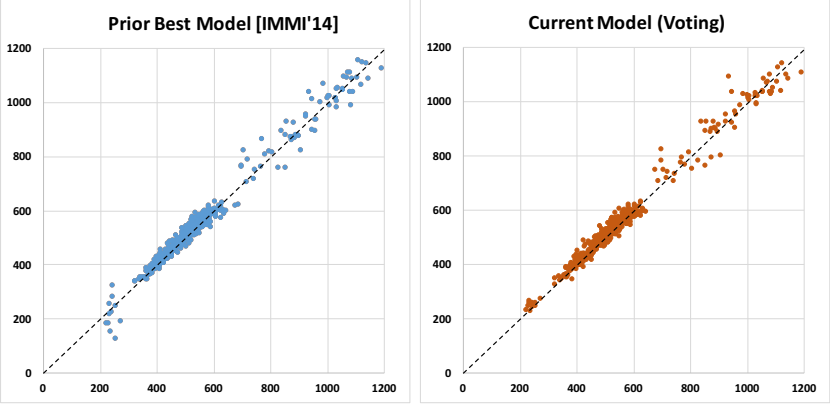

Figure 2: Scatter plots comparing the best model from [5] and the final model from the current study based on Voting scheme. The new model can be seen to perform significantly better in the low fatigue strength region of the plot where the old model had failed.

identified a subset of three processing attributes: THT (through hardening temperature), THQCr (cooling rate for through hardening), and Tt (tempering time). Subsequently we redid the modeling using the reduced set of nine attributes. Three best models were found to have statistically indistinguishable accuracy, and were thus combined using the Voting scheme, resulting in the following accuracy numbers: $R^{2}=0.9440, M A E=36.41 M P a, R M S E=44.14 M P a$.

Typically, data-driven predictive models are not simple equations and require the use of specialized software to make predictions. To make the forward predictive models developed in this work readily accessible for use by the materials science and engineering community, we have created an online steel fatigue strength predictor that can take as input the values of the reduced set of 9 attributes, and generate predictions of fatigue strength for a given steel. The reduced set of attributes was chosen for the tool so that the user does not have to enter too many attributes and still gets satisfactory accuracy, but the option of using the full calculator with all 25 attributes is also available. The screenshot of the calculator is depicted in the Appendix, and it is available online at http://info.eecs.northwestern. edu/SteelFatigueStrengthPredictor. 
Table 1: Predictive modeling algorithms explored in this work

\begin{tabular}{ll}
\hline Modeling Technique & Brief description \\
\hline Linear regression & A linear least-squares fit of the data with respect to the input features \\
Nearest-neighbor (IBk) & Uses the most similar instance in the training data for making predictions \\
Nearest-neighbor (KStar) & Uses entropy based function for distance instead of Euclidean distance \\
Artificial neural networks (MLP) & Uses hidden layer(s) of neurons to connect inputs and outputs, edge weights learnt using back propagation \\
Gaussian process & Generates data following multivariate Gaussian distribution to make predictions \\
Support vector machines & Based on the Structural Risk Minimization, constructs hyperplanes multidimensional feature space \\
Decision table & Constructs rules involving different combinations of attributes \\
Decision stump & A weak tree-based machine learning model consisting of a single-level decision tree \\
M5 model tree & A tree with linear regression function at the leaves \\
Random tree & Considers a randomly chosen subset of attributes \\
Reduced error pruning tree & Builds a tree using information gain/variance and prunes it using reduced-error pruning to avoid over-fitting \\
Random forest & An ensemble of multiple random trees \\
Additive regression & Enhances performance of a base regressor by fitting a model to the residuals left in previous iteration \\
Bagging & Builds multiple models on bootstrapped subsets of data to improve model stability by reducing variance \\
Random committee & Ensemble of randomizable base models run with different seeds and individual predictions averaged \\
Random subspace & Constructs multiple trees systematically by pseudo-randomly selecting subsets of features \\
Rotation Forest & Generates model ensembles based on feature extraction followed by axis rotations \\
Voting & Combines predictions from multiple models by taking average, maximum, minimum, median, etc. \\
\hline
\end{tabular}

Table 2: Top 10 modeling techniques (10-fold cross-validation results, best statistically indistinguishable results at $p=0.05$, and the techniques used in final Voting model are boldfaced)

\begin{tabular}{|c|c|c|c|c|c|c|c|c|c|}
\hline Modeling Scheme & $R$ & $R^{2}$ & $\begin{array}{l}M A E \\
(M P a)\end{array}$ & $\begin{array}{l}R M S E \\
(M P a)\end{array}$ & $\begin{array}{l}R A E \\
(\%)\end{array}$ & $\begin{array}{c}R R S E \\
(\%)\end{array}$ & $\begin{array}{c}\text { TrainTime } \\
\text { (s) }\end{array}$ & $\begin{array}{c}\text { TestTime } \\
\text { (s) }\end{array}$ & $\begin{array}{c}\text { ModelSize } \\
\text { (bytes) }\end{array}$ \\
\hline RotationForest_M5 & 0.9900 & 0.9801 & 18.74 & 26.50 & 14.76 & 14.44 & 1.1058 & 0.0094 & 716215 \\
\hline RotationForest_MLP & 0.9894 & 0.9789 & 18.97 & 27.00 & 15.00 & 14.76 & 3.4866 & 0.0102 & 664851 \\
\hline Bagging_MLP & 0.9895 & 0.9791 & 18.97 & 27.03 & 14.99 & 14.78 & 3.0486 & 0.0009 & 99700 \\
\hline AdditiveRegression_M5 & 0.9897 & 0.9795 & 19.05 & 26.66 & 15.01 & 14.54 & 0.3996 & 0.0003 & 44210 \\
\hline Bagging_M5 & 0.9890 & 0.9781 & 19.36 & 27.96 & 15.23 & 15.21 & 0.8039 & 0.0008 & 264058 \\
\hline M5 ModelTrees & 0.9893 & 0.9787 & 19.64 & 27.46 & 15.45 & 14.94 & 0.0885 & 0.0001 & 19684 \\
\hline NeuralNetworks (MLP) & 0.9881 & 0.9763 & 19.89 & 28.41 & 15.72 & 15.56 & 0.3652 & 0.0002 & 13616 \\
\hline RandomCommittee_MLP & 0.9877 & 0.9756 & 20.37 & 29.14 & 16.05 & 15.84 & 3.0811 & 0.0010 & 99424 \\
\hline AdditiveRegression_MLP & 0.9851 & 0.9704 & 20.94 & 32.27 & 16.49 & 17.56 & 3.5265 & 0.0009 & 99476 \\
\hline RandomCommittee_REPTree & 0.9874 & 0.9750 & 21.39 & 29.48 & 16.86 & 16.09 & 0.0637 & 0.0003 & 83087 \\
\hline
\end{tabular}

\section{CONCLUSION AND FUTURE WORK}

In this demonstration paper, we compared 40 different modeling configurations for predicting fatigue strength of steel alloys using a publicly available experimental database from NIMS, and obtained significantly more accurate models than prior models. The most accurate forward models both on the full dataset and on a reduced set of attributes were deployed in a publicly accessible online web-tool. The primary advantage of such a tool is ready access to fast and accurate forward models of PSPP relationships without having to do experiments/simulations, which can help identify promising candidates for further exploration. The presented workflow can be readily applied on other experimental and computational materials science data.

\section{ACKNOWLEDGEMENTS}

We are grateful to NIMS for making the MatNavi database [3] publicly available, and also to the authors of reference [6] for preprocessing the raw NIMS data and making it publicly available as supplementary data accompanying reference [5]. This work was performed under the following financial assistance award 70NANB14H012 from U.S. Department of Commerce, National Institute of Standards and Technology as part of the Center for Hierarchical Materials Design (CHi$\mathrm{MaD})$. The authors also acknowledge partial support from AFOSR award FA9550-12-1-0458.

\section{REFERENCES}

[1] Materials Genome Initiative for Global Competitiveness, June 2011; OSTP 2011.

[2] Materials Genome Initiative Strategic Plan, National Science and Technology Council Committee on Technology Subcommittee on the Materials Genome Initiative, June 2014.

[3] URL: National Institute of Materials Science, http://smds.nims.go.jp/fatigue/index_en.html, Accessed: March 31, 2016.

[4] A. Agrawal and A. Choudhary. Perspective: Materials informatics and big data: Realization of the "fourth paradigm" of science in materials science. APL Materials, 4(053208):1-10, 2016.

[5] A. Agrawal, P. D. Deshpande, A. Cecen, G. P. Basavarsu, A. N. Choudhary, and S. R. Kalidindi. Exploration of data science techniques to predict fatigue strength of steel from composition and processing parameters. Integrating Materials and Manufacturing Innovation, 3(8):1-19, 2014.

[6] B. P. Gautham, R. Kumar, S. Bothra, G. Mohapatra, N. Kulkarni, and K. A. Padmanabhan. More Efficient ICME through Materials Informatics and Process Modeling, pages 35-42. John Wiley \& Sons, Inc., 2011.

[7] M. Hall, E. Frank, et al. The weka data mining software: An update. SIGKDD Explorations, 11(1), 2009 . 


\section{Steel Fatigue Strength $\mathcal{P}_{\text {redictor }}$}

Disclaimer: The results from this tool are estimates based on data consisting of a set of experimental measurements. All results are provided for informational purposes only, in furtherance of the developers' educational mission, to complement the knowledge of materials scientists and engineers, and assist them in their search for new materials with desired properties. The developers may not be held responsible for any decisions based on this tool.

Welcome to the steel fatigue strength predictor. This tool is built using experimental data of more than 400 steels, available from the Japan National Institute for Materials Science (NIMS) MatNavi database. This database consists of processing and composition information of steels, along with the experimentally measured rotating bending fatigue strength (10 million cycles). Various data analytics techniques such as feature selection and regression modeling were used to obtain highly accurate fatigue strength prediction models using a small non-redundant set of six composition parameters and three processing parameters.

The tool estimates the fatigue strength of the steel based on the composition and processing parameters entered by the user. To obtain the fatigue strength prediction, please enter the attribute values below, and click the submit button.

\begin{tabular}{|ll|}
\hline Carbon (C) (wt \%) & 0.388 \\
\hline Silicon (Si) (wt \%) & 0.3 \\
\hline Phosphorus (P) (wt \%) & 0.016 \\
\hline Chromium (Cr) (wt \%) & 0.57 \\
\hline Copper (Cu) (wt \%) & 0.068 \\
\hline Molybdenum (Mo) (wt \%) & 0.07 \\
\hline
\end{tabular}

\begin{tabular}{|l|l|}
\hline Through Hardening Temperature $\left({ }^{\circ} \mathrm{C}\right)$ & 850 \\
\hline Cooling Rate for Through Hardening $\left({ }^{\circ} \mathrm{C} / \mathrm{hr}\right)$ & 8 \\
\hline Tempering Time (minutes) & 60 \\
\hline
\end{tabular}

Have more attributes to enter?

Use the full calculator instead!

Get Steel Fatigue Strength Prediction

Predicted fatigue strength of the given steel $=\mathbf{5 1 2 . 7 8 8} \mathbf{M P a}$
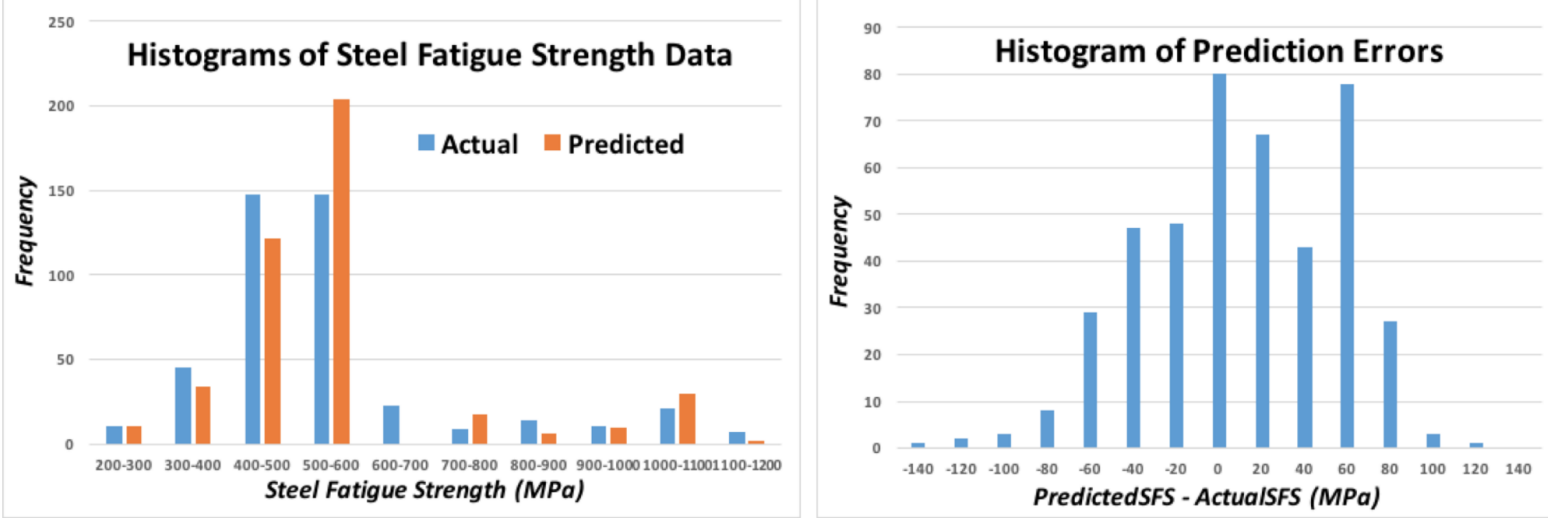

\section{Acknowledgements}

We are grateful to NIMS for making the raw data on fatigue steel strength publicly available, and also to the authors of reference [1] to preprocess the raw NIMS data and make it available as supplementary data accompanying reference [2].

\section{References:}

[1] B. P. Gautham, R. Kumar, S. Bothra, G. Mohapatra, N. Kulkarni and K. A. Padmanabhan, "More Efficient ICME through Materials Informatics and Process Modeling," Proceedings of the 1st World Congress on Integrated Computational Materials Engineering (ICME), 2011. [url]

[2] A. Agrawal, P. D. Deshpande, A. Cecen, G. P. Basavarsu, A. N. Choudhary, and S. R. Kalidindi, "Exploration of data science techniques to predict fatigue strength of steel from composition and processing parameters," Integrating Materials and Manufacturing Innovation, vol. 3, no. 8, pp. 1-19, 2014. [url]

Figure 3: A screenshot of the deployed steel fatigue strength predictor. 